\title{
Statistical physics of self-propelled particles
}

\author{
M.J.B. Hauser ${ }^{1, a}$ and L. Schimansky-Geier ${ }^{2, b}$ \\ 1 Biophysics Group, Institute of Experimental Physics, Otto von Guericke University \\ Magdeburg, Universitätsplatz 2, 39106 Magdeburg, Germany \\ 2 Department of Physics, Humboldt University at Berlin, Newtonstr. 15, 12489 Berlin, \\ Germany
}

Self-propelled particles (SPP) are a modern topic of interdisciplinary research at the frontier between physics and biology. These entities take up energy from their environment and convert (a part of) it into motion. This ability to move autonomously enables them to generate a new variety of singular properties and of collective dynamical behaviors. Autonomous motion and the behavioral patterns associated to SPPs are inherent to many living objects but also to some engineered particles and constructs.

Currently, various aspects of the physics of self-propelling particles are in the focus of scientific research. They encompass, among others, the mechanisms that bring about autonomous motion, the description of the types of motion, as well as the role played by fluctuations in the emergence of novel behaviors. Currently, the research on SPP points to three major directions, namely (i) the motion and transport of individual self-propelled objects, (ii) the motion of active particles in external and/or self-generated fields, and (iii) the collective behavior of a group of particles that act upon each other via binary interactions.

Recently, an increasing number of scientific studies on SPP is observed with a main focus on the determination of the statistical properties of the motion. This is the case in studies of contemplative motion, i.e., motion where the particle is free to move without any confinements or gradients acting upon them. One of the main objectives of such studies is to assess and understand the role of fluctuations in the motion of SPPs. The statistics of such free migration may provide useful information on the efficiency of the different types of motion.

Many systems, however, move either in an external gradient field, or even produce their own gradient field that, in turn, affects the motion of the SPPs. Such types of interplay may be found in biological systems where a cell or organism relays a chemoattractant, pheromone, or the like, but also in non-living systems, where the swimming of a particle may induce hydrodynamic fields that affect the swimming of the other particles. Such interactions may lead to motions, the statistics of which differ considerably from those found for free migration.

Finally, systems of SPPs that are coupled to each other by binary interactions may generate interesting patterns of group dynamics, for instance, as seen in herds of sheep, schools of fish, or flocks of birds. Such collective motion may provide huge advantages for the members of the group, which adds to the scientific interest in such

\footnotetext{
a e-mail: marcus.hauser@ovgu.de

b e-mail: alsg@physik.hu-berlin.de
} 
systems and their organization. Here, again, the role of fluctuations on the collective dynamics is a hot topic of research.

The current Topical Issue provides a collection of contributions devoted to the study of several situations of single particles and of ensembles of them. The unifying viewpoint for the kinetic modelling of single particles as well as of many particle systems is given by both: By statistical physics and its successful theoretical methods and models on the one hand, and by a variety of experimental tools at the other. As elaborated in the contributions of this Issue, the modelling of the spatio-temporal behavior as non-equilibrium processes requires new tools in the theory of nonlinear stochastic dynamics as well as novel experimental techniques for in vivo observations. Therefore, the concern of physicists with these assorted novel situations in mostly living matter is of highly interdisciplinary benefit.

This Topical Issue contains mini-reviews and original papers that cover various timely topics of research on self-propelled particles. In particular, the following topics are addressed in the present Topical Issue:

The first contribution, authored by Vestergaard et al. [1], examines how the motility parameters of a SPP can be estimated from experimental data, in particular, in cases where empirical data analysis is difficult, that is, when the trajectories are short and when there is only a limited number of them. Considering the case when particles perform ordinary Brownian motion with a linear time-dependence of the mean squared displacement, the authors thoroughly discuss different sources of errors and provide the best estimator for diffusion coefficients.

Similar methods are applied in the following study by Götz et al. [2], who investigated the impact of the intracellular architecture and cytoskeleton dynamics on intracellular transport. To this purpose, the local mean squared displacement of the motion of silica particles engulfed by the slime mold Dictyostelium discoideum were studied experimentally. Using analysis methods the authors find active transport, subdiffusive, diffusive and superdiffusive particle motion.

Raatz and co-authors [3] analyze swimming patterns of the bacterium Pseudomonas putida in a variety of confined environments. They describe a systematic approach using proven experimental (microfluidic) technologies and a data analysis algorithm to characterize the swimming motility of $P$. putida by sequences of directional runs separated by turning events. The authors discuss the hydrodynamic effect of interfaces and obstacles on the swimming patterns, and demonstrate how increasing spatial confinement leads to changes in turning angle statistics and mean run lengths of the bacteria.

The motility of amoeboid cells of the slime mold Physarum polycephalum was studied experimentally by Rodiek and Hauser [4]. Analysis of the trajectories and of their mean square displacements reveal two characteristic types of behavior that depend on the time intervals considered: Free migration of cells similar to persistent random motion and a motility which is due to changes in the cell shape induced by the peristaltic pumping of protoplasm though the cell. During free migration, superdiffusion was also observed and the typical velocity distributions from freely migrating cells show an asymmetric component. High propagation velocities are correlated with both, episodes of straight motion and an elongated cell shape. The mean squared displacement of trajectories where cells avoided crossing their own slime trails were compared to those of freely migrating cells.

Romanczuk et al. [5] report on the velocity distributions of the swimming algae Euglena gracilis in a microfluidic channel and interpret the data. The observed velocity distributions are rationalized through a theory based on active Brownian motion with active fluctuations, showing good agreement. Key point of the applied concept of active fluctuations is the fact that force fluctuations point along the heading direction of propulsion. The fluctuations arise due to the random internal performance 
of the propulsive motors, hence, originating in the noise inherent in the propulsive mechanism.

Solon et al. [6] provide a theoretical comparison of "active" and "run and tumble" movements. In both cases, the analyses are based on the kinetic description by means of master equations and equations for the moments of the probability density functions. The authors investigate the behavior of single particles in both external fields and confinements. Furthermore, the interaction of particles was also studied. A wealth of material is provided, which also includes a comprehensive description of the technical details of the applied approach. Last, but not least, the crossover from a particle-oriented description to a field description with density-dependent velocity is also elaborated.

Hernàndez-Navarro et al. [7] review driven motion of colloids in anisotropic matrices like nematic liquid crystals. The driving mechanism of the colloids is based on the principle of nonlinear electrophoresis which is mediated by the asymmetry in the structure of the defects generated by inclusions in the host's elastic matrix. The authors discuss various types of individual and collective motion of charged colloids, which were induced by electric fields.

The motion of two wedge objects in a bath of self-propelled rods is studied in the contribution by Kaiser et al. [8]. The wedges are displaced when pushed by the self-propelled rods while at all times their orientation is conserved. In experiments, this situation is controlled due to the presence of a magnetic field acting upon them.

As a simple model of SPPs in heterogeneous environments, Chepizhko and Peruani [9] study self-propelled particles interacting with obstacles. It is shown that for a sufficiently developed spatial heterogeneity, the well-documented high-density, high-ordered propagating bands that emerge in homogeneous space disappear. The ordered phase does not exhibit long-range order, as in homogeneous systems, but rather quasi-long-range order. That is, the SPP system becomes disordered in the thermodynamic limit. For finite-size systems an optimal noise value exists that maximizes the order.

Ihle [10] discusses the hydrodynamic limit of the Vicsek model for collective motion. The analysis is based on an exact equation for the N-particle distribution function. Hydrodynamic equations are obtained in two cases by using a mean-field approximation and by a dimensional reduction via elimination of fast variables.

As an amendment to the pure Vicsek model, Großmann et al. [11] show that added particles provide for additional alignment rules. Apart from a repulsion-like interaction at very short distances, particles show parallel alignment interactions at low separation distances, whereas at a higher separation, they feature antiparallel alignments. Finally, highly separated particles do not interact at all. These competing effects lead to a rich variety of structures and patterns on the collective level, for example clustered structures and apparently turbulent regimes.

The general question how to describe a fluid that contains SPPs or bacteria using an effective Navier-Stokes-like equation is addressed by Slomka and Dunkel [12]. Typically, two coupled equations are derived, both for the fluid velocity field and for the active component. The authors focus on an effective one-component description but add higher-order terms into the stress tensor. The resulting generalized NavierStokes equation has not been studied so far. The authors perform a stability analysis, provide a numerical solution in two spatial dimensions, and analyze the scaling of the energy spectrum.

Romensky and Lobaskin [13] present a numerical study of a two-dimensional active Brownian particle model confined to a narrow channel. The authors investigate dynamic hysteresis effects of the polar order parameter in an external oscillating field. Main results are the emergence of non-universal scaling exponents for the hysteresis 
loop domain and how the limiting values of the hysteresis loop can be predicted by a generic model of dynamic hysteresis.

A special kind of confinement in $d$-dimensions is also studied by Großmann et al. [14], who propose a geometric perspective to describe the motion of self-propelled particles moving at constant speed. If a vector conveys such a confinement, the direction of motion of the SPP performs a random walk on a $(d-1)$-dimensional manifold. It is shown that the SPP performs isotropic diffusion in the $d$-dimensional space when the manifold corresponds to a hypersphere. In contrast, when the manifold corresponds to a deformed hypersphere (e.g. an ellipsoid), the self-propelled particles exhibit anisotropic diffusion.

The present Topical Issue closes with a contribution dealing with a different but highly important question concerning the efficiency of self-propelled particles, in particular, of molecular motors. Ebeling [15] shows that in a narrow domain of parameters, SPPs may indeed reach high efficiencies. However, it also demonstrated that such high-efficiency situations become highly improbable in the presence of noise.

The editors thank all the authors for their valuable contributions and cooperation. We wish to extend our thanks to all referees for their thorough and prompt refereeing of the manuscripts. Special thanks to Ms. Sabine Lehr (Springer) and Mrs. Isabelle Houlbert (EDP Sciences) for the assistance, encouragement and prompt responses.

We are grateful to the WE-Heraeus Foundation for the financial support and help with the organization of the 565th WE-Hereaus Seminar on "Statistical Physics of Self-Propelling Particles: Theory and Experiments", which laid the seed for the present Topical Issue.

\section{References}

1. C.L. Vestergaard, J.N. Pedersen, K.I. Mortensen, H. Flyvbjerg, Eur. Phys. J. Special Topics 224(7), 1151 (2015)

2. M. Götz, K.F. Hodeck, P. Witzel, A. Nandi, B. Lindner, D. Heinrich, Eur. Phys. J. Special Topics 224(7), 1169 (2015)

3. M. Raatz, M. Hintsche, M. Bahrs, M. Theves, C. Beta, Eur. Phys. J. Special Topics 224(7), 1185 (2015)

4. B. Rodiek, M.J.B. Hauser, Eur. Phys. J. Special Topics 224(7), 1199 (2015)

5. P. Romanczuk, M. Romensky, D. Scholz, V. Lobaskin, L. Schimansky-Geier, Eur. Phys. J. Special Topics 224(7), 1215 (2015)

6. A.P. Solon, M.E. Cates, J. Tailleur, Eur. Phys. J. Special Topics 224(7), 1231 (2015)

7. S. Hernàndez-Navarro, P. Tierno, J. Ignés-Mullol, F. Sagués, Eur. Phys. J. Special Topics 224(7), 1263 (2015)

8. A. Kaiser, A. Sokolov, I.S. Aranson, H. Löwen, Eur. Phys. J. Special Topics 224(7), $1275(2015)$

9. O. Chepizhko, F. Peruani, Eur. Phys. J. Special Topics 224(7), 1287 (2015)

10. T. Ihle, Eur. Phys. J. Special Topics 224(7), 1303 (2015)

11. R. Großmann, P. Romanczuk, M. Bär, L. Schimansky-Geier, Eur. Phys. J. Special Topics 224(7), 1325 (2015)

12. J. Slomka, J. Dunkel, Eur. Phys. J. Special Topics 224(7), 1349 (2015)

13. M. Romensky, V. Lobaskin, Eur. Phys. J. Special Topics 224(7), 1359 (2015)

14. R. Großmann, F. Peruani, M. Bär, Eur. Phys. J. Special Topics 224(7), 1377 (2015)

15. W. Ebeling, Eur. Phys. J. Special Topics 224(7), 1395 (2015) 